\title{
SUFFICIENT CONDITIONS FOR A CLOSED SET TO LIE ON THE BOUNDARY OF A 3-CELL
}

\author{
L. D. LOVELAND
}

Let $F$ be a closed subset of a 2 -sphere $S$ in $S^{3}$, and let $V$ be a component of $S^{3}-S$. Using some results and proofs from [7] and [8] we give several conditions that are sufficient for $F$ to lie on the boundary of a 3-cell. One such condition is Property $(*, F, V)$ which is defined in [7]. Property $\left({ }^{*}, F, V\right)$ roughly means that the polyhedral approximation $S^{\prime}$ to $S$ obtained using Bing's Side Approximation Theorem [2] can be chosen such that $S^{\prime}$ lies "almost" in $V$ and $S \cap S^{\prime}$ lies in the union of a finite collection of disjoint small disks in $S-F$.

Property $\left({ }^{*}, F, S\right)$ is defined in [7] to mean that Property $\left({ }^{*}, F, V\right)$ is satisfied for each component $V$ of $S^{3}-S$. The author [7] established a conjecture made by Gillman [5] and then used some of Bing's techniques [1] to show that $F$ lies on a tame 2-sphere if Property $(*, F, S)$ is satisfied. Unfortunately the following theorem was not included in [7] and is not a direct consequence of any of the results there.

Theorem 1. If $\epsilon>0, F$ is a closed subset of a 2-sphere $S$ in $S^{3}$, and $V$ is a component of $S^{3}-S$ such that Property (*, $\left.F, V\right)$ is satisfied, then there is a continuum $M$ on $S$ and a null sequence $\left\{D_{i}\right\}$ of disjoint $\epsilon$-disks on $S$ such that (1) $M=S-\cup$ Int $D_{i}$, (2) Property (*, $\left.M, V\right)$ is satisfied, (3) $F \subset M-\cup D_{i}$, and (4) $M$ lies on the boundary of a 3-cell.

Lister [6] made use of Theorem 1 in showing that $h(F)$ lies on the boundary of a 3-cell if $F$ and $S$ satisfy Property $(*, F, V)$ and $h$ is a homeomorphism of $S \cup V$ into $S^{3}$. Burgess and Loveland [4] used Theorem 2, which is a consequence of Theorem 1, in giving conditions for $F$ to lie on the boundary of a 3-cell.

A proof for Theorem 1 can be obtained following the same general pattern as in the proof of Theorem 6 of [7] provided we have a "onesided" version of Gillman's conjecture to replace Theorem 3 of [7]. Such a version of the conjecture is stated and proved here as Lemma 1. The proof given for Lemma 1 is based on consequences of a "twosided" version of the conjecture (Theorem 3 of [7]) given in [7]. We feel that to prove Theorem 1 here would be to a large extent a repeat of $\$ 2$ of [7]. For this reason we prove only Lemma 1 and then we rely on [7] for an indication of how Lemma 1 is used to obtain a proof for Theorem 1.

Received by the editors January 3, 1967. 
Levma 1. Suppose $F$ is a closed subset of a 2-sphere $S$ in $E^{3}$ such that Property $\left({ }^{*}, F\right.$, Int $\left.S\right)$ is satisfied. There is a positive number $\epsilon$ such that if h is a homeomorphism of $S$ into $E^{3}$ which moves each point less than a distance $\epsilon$ and which is the identity on the complement of a finite collection $D_{1}, D_{2}, \cdots, D_{n}$ of disjoint disks in $S-F$, then Property $\left({ }^{*}, F\right.$, Int $\left.h(S)\right)$ is also satisfied.

Proof. Let $p \in$ Int $S, q \in$ Ext $S$, and let $\epsilon$ be a positive number less than the distance between $S$ and $\{p, q\}$. Suppose $h$ is a homeomorphism and $D_{1}, D_{2}, \cdots, D_{n}$ is a finite collection of disks as in the statement of Lemma 1 . We denote $h\left(D_{i}\right)$ by $E_{i}$ for each $i$, and we let $h(S)=S^{\prime}$. From the restriction on $\epsilon$ we see that $p \in \operatorname{Int} S^{\prime}$ and $q \in$ Ext $S^{\prime}$.

Let $x$ be a point in $F$, and let $N$ be an open set containing $x$ such that $N \cap\left(\mathrm{U}\left(E_{i} \cup D_{i}\right)\right)=\varnothing$. It follows from Theorem 11 of [7] that Property ( ${ }^{*}, F$, Int $S$ ) implies there is an open set $U$ containing $x$ such that each simple closed curve in $U \cap$ Int $S$ can be shrunk to a point in $N-F$. With no loss in generality we assume that $U$ is the interior of a 2 -sphere. Let $J$ be a simple closed curve in $U \cap$ Int $S^{\prime}$. We will show that $J \subset \operatorname{Int} S$, so that $J$ can be shrunk to a point in $N-F$. The compactness of $F$ will then insure the following uniform property:

Property $\left(A, F\right.$, Int $\left.S^{\prime}\right)$ : For each $\alpha>0$ there is a $\delta>0$ such that each simple closed curve of diameter less than $\delta$ which lies in Int $S^{\prime}$ can be shrunk to a point in an $\alpha$-subset of the complement of $F$.

An argument similar to the proof of Theorem 2 of [7] shows that we could have begun by assuming that the set of diameters of the components of $F$ has a positive lower bound. Then the proof of Theorem 10 of [7] insures that Property $\left(*, F\right.$, Int $\left.S^{\prime}\right)$ is satisfied.

All that remains is to show that $J \subset$ Int $S$. We suppose $J$ contains a point $t$ in Ext $S$; and we let $W$ be an arc in $U$ from $t$ to some point $y$ in $S$ such that $W \cap\left(S^{\prime} \cup S\right)=\{y\}$. It follows from Theorem 14 of [2] that there is an $\operatorname{arc} X$ from a point $r$ in $S-\cup D_{i}$ to $p$ such that $X-\{r\}$ does not intersect $S \cup\left(\cup E_{i}\right)$. This means that $X-\{r\} \subset$ Int $S \cap \operatorname{Int} S^{\prime}$. If $r \neq y$ we use the fact that Int $S$ is 0 -ulc to construct an arc $Y$ from a point $r^{\prime}$ of $X$ to the point $y$ such that $Y-\{y\} \subset$ Int $S \cap$ Int $S^{\prime}$ and $Y \cap X=\left\{r^{\prime}\right\}$. Let $X^{\prime}$ be the subarc of $X$ from $p$ to $r^{\prime}$. Now the arc $A=X^{\prime} \cup Y \cup W$ pierces $S$ at $y$. This means that there is a disk $D$ in $S \cap U$ such that $A$ pierces $D$. Since $D \subset S^{\prime}, A$ also pierces $S^{\prime}$ at $y$. This means that $A$ intersects each complementary domain of $S^{\prime}$, yet $A-\{y\} \subset$ Int $S^{\prime}$. A similar argument (where $X^{\prime}$ is replaced by $X$ ) yields the same contradiction in the case where $r=y$. This completes the proof. 
If $F$ is a closed subset of a 2 -sphere $S$ in $S^{3}$ and $V$ is a component of $S^{3}-S$, we define Property $(C, F, V)$ to mean that for each $\epsilon>0$ there is a $\delta>0$ such that each unknotted simple closed curve that has diameter less than $\delta$ and that lies in $V$ can be shrunk to a point in an $\epsilon$-subset of $S^{3}-F$. Notice that Property $(A, F, V)$, as defined in the proof of Lemma 1, implies $(C, F, V)$. The proof of Theorem 10 of [7] shows that $(A, F, V)$ implies Property $(*, F, V)$ if the diameters of the components of $F$ have a positive lower bound. Actually the proof of Theorem 10 of [7] shows that $(C, F, V)$ implies $(*, F, V)$ under the same restriction on the components of $F$, since the only simple closed curves that needed to be shrunk to a point were on polyhedral 2spheres. Theorem 2 is a consequence of Theorem 1 and these observations.

Theorem 2. If $F$ is a closed subset of a 2-sphere $S$ in $S^{3}$ such that the diameters of the components of $F$ have a positive lower bound and Property $(C, F, V)$ is satisfied for some component $V$ of $S^{3}-S$, then $F$ lies on the boundary of a 3-cell.

Theorem 3. Suppose F is a closed subset of a 2-sphere $S$ in $S^{3}$ such that the diameters of the components of $F$ have a positive lower bound. Each of the following is sufficient for $F$ to lie on the boundary of a 3-cell:

(a) $F$ can be locally spanned from $V$; that is, for each $\epsilon>0$ and for each point $p \in F$ there are disks $R$ and $D$ such that $p \in \operatorname{Int} R \subset S$, $\operatorname{Bd} D=\operatorname{Bd} R, D-\operatorname{Bd} D \subset V$, and $\operatorname{diam}(D \cup R)<\epsilon$.

(b) $F$ can be locally spanned in $V$ on tame simple closed curves; that is, for each $\epsilon>0$ and for each $p \in F$ there is an $\epsilon$-disk $R$ such that $p \in$ Int $R$ $C S, \mathrm{Bd} R$ is tame, and for each $\alpha>0$ there is an $\epsilon$-disk $D$ in $V$ so that $\mathrm{Bd} R$ can be shrunk to a point in $D \cup($ an $\alpha$-neighborhood of $\mathrm{Bd} R)$.

(c) For each $p \in F$ and for each $\epsilon>0$ there is a 2-sphere $S^{\prime}$ of diameter less than $\epsilon$ such that $p \in \operatorname{Int} S^{\prime}$ and $S \cap S^{\prime}$ is a continuum satisfying Property (*, $\left.S \cap S^{\prime}, V\right)$.

The proofs given for Theorems 8 and 11 of [8] need only slight modification to show that (b) and (c) each imply Property $(*, F, V)$. The proof of Theorem 7 of [3] can be adjusted as suggested in [8, p. 364] to show that (a) also implies Property (*, $F, V)$. Thus Theorem 3 also follows from Theorem 1 .

\section{REFERENCES}

1. R. H. Bing, Each disk in $E^{3}$ contains a tame arc, Amer. J. Math. 84 (1962), 583590.

2. - Approximating surfaces from the side, Ann. of Math. 77 (1963), 145192. 
3. C. E. Burgess, Characterizations of tame surfaces in $E^{3}$, Trans. Amer. Math. Soc. 114 (1965), 80-97.

4. C. E. Burgess and L. D. Loveland, Sequentially 1-ULC surfaces in $S^{3}$, Notices Amer. Math. Soc. 14 (1967), 96.

5. David S. Gillman, Side approximation, missing an arc, Amer. J. Math. 85 (1963), 459-476.

6. F. M. Lister, Simplifying intersections of disks in Bing's Side Approximation Theorem, Pacific J. Math. 22 (1967), 281-295.

7. L. D. Loveland, Tame subsets of spheres in $E^{3}$, Pacific J. Math. 19 (1966), 489517.

8. - Tame surfaces and tame subsets of spheres in $E^{8}$, Trans. Amer. Math. Soc. 123 (1966), 355-368.

Utah State University 